\title{
Insulin Autoimmune Syndrome (Hirata Disease): Case Report in a Caucasian Patient with New-Onset Diabetes
}

\author{
Maurizio Sudano ${ }^{1}$, Federica Turchi ${ }^{1}$, Paolo Soss ai ${ }^{2, *}$ \\ ${ }^{1}$ Diabetes and Metabolic Diseases Unit, General Hospital, I - 61029, Urbino, Italy \\ ${ }^{2}$ Center of Clinical Research, School of Medicinal and Health Products Sciences, University of Camerino, I- 62032, Camerino, Italy
}

\begin{abstract}
An unusual cause of spontaneous hypoglycemia is Insulin Autoimmune Syndrome (or Hirata Disease), characterized by high levels of insulinemia and circulating autoantibodies to insulin in subjects without prior insulin administration. In Western Countries less than 70 cases have been published so far. Here we report a case where IAS was the prodromical stage of new onset type 1 diabetes. HLA typing in IAS was rarely reported in Western cases, here we also discuss the peculiar HLA pattern of our patient.
\end{abstract}

Keywords Hirata Disease, Insulin Autoimmune Syndrome, Autoimmunity, Hypoglycemia, HLA, Autoantibodies, Insulin, Type 1 Diabetes

\section{Introduction}

Insulin Autoimmune Syndrome (IAS) or Hirata Disease (HD), was first described by Hirata in 1970[1] and is characterized by spontaneous hypoglycemic episodes, a high titer of insulin autoantibodies and increased levels of immunoreactive insulin in patients not previously treated with insulin or oral hypoglycemic agents. There is a significant genetic predisposition to IAS as suggested by its association with specific HLA class II alleles, and it is often associated with previous exposure to drugs with a sulfhydryl group in their chemical structures such as methimazole or captopril. The majority of cases are reported in Japan, where IAS is the third leading cause of hypoglycemia (325 patients diagnosed at the end of 2007)[1]. The syndrome is rare in Caucasian/non-Japanese populations: 60 cases in Caucasians and 20 cases in East Asians have been reported to date[2][3]. To the best of our knowledge, the present case is the third reported in Italy (although the subject is not of Italian origin) and the first with HLA typing[4][5].

\section{Case Report}

A 25-year old Caucasian male originally from Eastern Europe (Bulgaria), presented to the Emergency Department of the General Hospital of Urb ino with dizziness, sweating and fine tremors. These symptoms were not accompanied by

\footnotetext{
* Corresponding author:
}

paolosossai@libero.it (Paolo Sossai)

Published online at http://journal.sapub.org/cmd

Copyright @ 2012 Scientific \& Academic Publishing. All Rights Reserved nausea, vomiting or headache. He reported a one-month history with similar recurrent episodes not strictly related to meals. His past medical history was significant only for spinal surgery due to severe spondylolisthesis. He had not previously taken any medications except for short-term therapy with steroids before spinal surgery. He denied any history of alcoholism or drug addiction.

On admission the patient appeared alert and oriented to person and time. His vital signs were stable. His Body Mass Index (BMI) was 20.2. His plasmatic glycemia was 2.94 $\mathrm{mmol} / \mathrm{L}(53 \mathrm{mg} / \mathrm{dl})$ and he recovered fully after an intravenous infusion of glucose. After discharge he suffered from hypoglycemic episodes almost every day, occurring both in a fasting state and 2-3 hours after meals. According to the Whipple triad[6] his symptoms were relieved by intake of food rich in carbohydrates. About five months after discharge, early investigations showed normal fasting glycemia $(81 \mathrm{mg} / \mathrm{dl}, 4.49 \mathrm{mmol} / \mathrm{L})$ and insulinemia $(3.4$ $\mu \mathrm{U} / \mathrm{ml}$, normal range 1.9-23). He was subsequently referred to us for closer examination. The oral glucose challenge test (OGTT) was positive for diabetes mellitus, as shown in Table 1, yet glycated hemoglobin was still with in the normal range (5.4\%, range. $\leq 6.2)$. Unlike the previous test, fasting insulinemia was very high $(181.1 \mu \mathrm{U} / \mathrm{ml})$.

Table 1. OGTT

\begin{tabular}{|c|c|c|c|c|c|}
\hline Time & $0 \mathrm{~min}$ & $30 \mathrm{~min}$ & $60 \mathrm{~min}$ & $90 \mathrm{~min}$ & $120 \mathrm{~min}$ \\
\hline $\begin{array}{c}\text { glycemia } \\
(\mathrm{mg} / \mathrm{dl})\end{array}$ & 93 & 249 & 324 & 230 & 78 \\
\hline
\end{tabular}

An abdominal ultrasonography and Magnetic Resonance Imaging (MRI) were done in order to investigate the possibility of insulinoma, but both tests were negative. We 
decided to perform a supervised 48h fast test in an attempt to show an inappropriate insulin incretion. Unfortunately, due to a technical error, the test was discontinued after $12 \mathrm{hr}$, yet the results were significant as shown in table 2 .

Table 2. Fast Test

\begin{tabular}{|c|c|c|c|}
\hline Time & $\begin{array}{c}\text { Insulinemia } \\
(\mu \mathrm{U} / \mathrm{ml})\end{array}$ & $\begin{array}{c}\text { Glycemia } \\
(\mathrm{mg} / \mathrm{dl})\end{array}$ & $\begin{array}{c}\text { C Peptide } \\
(\mathrm{ng} / \mathrm{dl})\end{array}$ \\
\hline $7 \mathrm{am}$ & 102.9 & 80 & 6.3 \\
\hline $12 \mathrm{~mm}$ & 78.2 & 73 & 5.6 \\
\hline $4 \mathrm{pm}$ & 59.6 & 77 & 10.4 \\
\hline $8 \mathrm{pm}$ & 48.3 & 70 & 13.1 \\
\hline $12 \mathrm{pm}$ & 38.7 & 63 & 6.2 \\
\hline
\end{tabular}

Additional tests revealed high levels of antiglutamic acid decarboxylase (GADA) antibodies (16 IU/ml normal range $\leq 5$ ) and antiinsulin antibodies (IAA, $16.5 \mathrm{IU} / \mathrm{ml}$, normal range $\leq 2.4$ ).

On the basis of all tests performed, we diagnosed HD. Since the association of IAS with specific genetic loci is well known[7], we took blood samples for HLA Class II typing. The results are shown in Table 3.

Table 3. HLA Class II Typing

\begin{tabular}{|c|c|}
\hline DRB1 $*$ 04:03 & DRB1*15 \\
\hline DQA1 $01: 03$ & DQA1 $03: 01$ \\
\hline DQB1*03:02 & DQB1*05 \\
\hline
\end{tabular}

Note: HLA-DRB1*04:06 not present

\section{Discussion}

The patient presented to the Emergency Department with symptoms suggesting recurrent 'hypoglycemic crisis'. The symptoms of hypoglycemia are classified as neurogenic (adrenergic) or neuroglycopenic. Neurogenic symptoms, caused by the stimulation of sympathetic nervous system, include sweating, palpitation, fine tremors, anxiety and hunger. Usually, adrenergic symptoms precede the neuroglycopenic symptoms and can be useful as early warnings for hypoglycaemic patients. Neuroglycopenic symptoms - due to decreased cerebral glucose availability include confusion, concentration difficult ies, irritability, and focal cerebral impairment.

The differential diagnosis for hypoglycemia is quite complex and must take into account several pathological conditions (Table 3). Drugs, including diabetes mellitus-con trolling medications, and alcohol are considered to be the leading causes of hypoglycemia. Other well-known causes of hypoglycemia include endogenous hyperinsulinism, such as insulinoma, critical illnesses and endocrine deficiencies.

The general condition and routine tests of our patients showed no evidence for coexisting critical diseases. High concentrations of C-Peptide were coexisting with raised insulin levels: this finding allowed us to exclude hypoglyca emia fromself-administered insulin (hypoglycaemia factitia). On the other hand, insulino ma is the most common cause of hypoglycemia due to endogenous hyperinsulinism. At the onset of an insulinoma, hypoglycemic crises occur in fasting state but, with time, patients develop persistent hypoglycaemia. However, we did not found any evidence of tumours from MRI or ultrasound scan. Besides, as we will discuss herein, fast test was not significant for insulino ma.

Table 3. Differential diagnosis for hypoglycemia

\begin{tabular}{l}
\hline Drugs \\
Insulin, sulfonylureas, ethanol, pentamidine, quinine, salicylates, \\
sulfonamides, \\
Endogenous hyperinsulinism \\
Insulinoma \\
Other $\boldsymbol{\beta}$ cell disorders \\
Autoimmune (autoant ibodiesto insulin, insulin receptor, $\beta$ cell?) \\
Ectopic insulin secretion \\
Critical illnesses \\
Hepatic, renal, or cardiac failure \\
Sepsis \\
Starvation and inanition \\
Endocrine deficiencies \\
Hypopituitarism \\
Non- $\beta$-cell tumours \\
Fibrosarcoma, mesothelioma, rhabdomyosarcoma, liposarcoma,, \\
hepatoma, adrenocorticaltumours, carcinoid, leukemia, lymphoma, \\
melanoma, teratoma \\
Disorders of infancy or dhildhood \\
Infants of diabetic mothers (hyperinsulinism) \\
Persistent hyperinsulinemic hypoglycemia of infancy \\
Inherited enzyme defects \\
Postprandial \\
Reactive (after gastric surgery) \\
Ethanol-induced \\
Factitious \\
Insulin, sulfonylureas
\end{tabular}

IAS (or HD) is characterized by the presence of autoantibodies to native insulin (in most cases with a polyclonal pattern), and hypoglycemic crises are held to be caused by the erratic dissociation of insulin-antibody complexes. It has been hypothesized that after a meal the raised plasmatic glucose concentration evokes the release of insulin, which in most part becomes bound to autoantibodies and thus is not biologically available. As a result, the postprandial hyperglycemia is not adjusted and keep on stimulating insulin-secretion. During post-prandial phase, as glycemia declines, the kinetics of the insulin-antibody interaction shifts to favour the dissociation of insulin-antibo dy complexes, resulting in inappropriately high serum free insulin concentrations in the presence of decreasing glucose concentrations, and consequent hypoglycemia[8].

Despite its well-known genetic background the pathological trigger for IAS has not been elucidated yet. Typically, as in our case, affected patients experience hypoglycemic crises both in the fasting and postprandial state. In IAS, insulin levels are inappropriately high for a given glucose concentration, but unlike insulinoma, are usually suppressed by prolonged fasting. Despite the accidental interruption, the fast test in our patient revealed a clear trend for insulin to be reduced after a few hours. The ratio of insulin to glucose is about 1 and increases with fasting in patients with insulinoma[9]: In our patient, after 
12h of fasting the insulin/glucose ratio was about 0.6 with an evident lowering trend.

As expected, antiinsulin antibodies were elevated, yet in this case IAS was diagnosed along with diabetes mellitus. Broadly speaking, type 1 diabetes mellitus (T1DM) is associated with additional autoimmune diseases, which are associated with the production of organ-specific autoantibo dies[12], however a few other cases with the association T1DM/IA S have been published[5][10]. Here, along with IAA, high levels of GADA were found in our patient: the concurrent presence of IAA and GADA in a subject with a positive OGTT is highly suggestive of new-onset T1DM.

T1DM has a prodromal stage of islet autoimmunity, and affected children develop up to four different antibody classes[11]. We did not perform tests for Insulinoma Antigen-2 (IA-2A) or ZnT8 transporter autoantibodies (ZnT8A).

Yet, the presence of IAA is suggestive of T1DM in its typical presentation, given that in Latent Autoimmune Diabetes of the Adult (LADA) they are usually absent.

HLA Class II typing revealed the presence of DRB1*04:03 DQB1*03:02 and DQA 1*03:01 alleles in our patient. Japanese population shows the highest frequency of IAS in the world and patients present with DRB1*0403 (as in our patient) and DRB1*0406 DRB1*0407 alleles as well (not present in our case). However, DRB1*0403 is considered the "ancestral” allele of DRB1*0406[7].

Our patient also possesses DQB1*03:02 and DQA 1*03:0 1 alleles, found in East Asia IAS cases outside Japan.

It is worth pointing out that our patient was born in Bulgaria: from a genetic point of view, Bulgarian population shows a predominant Mediterranean HLA Class I pattern, but some rare haplotypes suggest the influence of other groups such Asians, Armenian and Turkish[12]. Unfortunately, we was not able to get a comprehensive familiar history from the patient.

It must be emphasized that T1DM is often associated with the presence of additional autoimmune diseases with many organ system involved[13]. In our patient IAS was an epiphenomenon of immunity derangement occurring during the development of Type 1 diabetes.

Interestingly, on follow-up the patient's C peptide dropped to $0.7 \mathrm{ng} / \mathrm{dl}$, along with IAA (decreased to 5.2 $\mathrm{IU} / \mathrm{ml}$ ). At the same time the GADA antibodies disappeared, as often seen in the prodromal stage of Type 1 diabetes[14].

The patient was prescribed a fractionated, normocaloric diet (5 meals/day), and experienced a significant relief of symptoms, perhaps also due to IAA levels being reduced. The patient, despite the low level of C peptide, is still in good condition 12 months after the ons et of symptoms, and so far, has not required insulin therapy.

\section{REFERENCES}

[1] Hirata Y, Ishizu H, Ouchi N, et al. Insulin autoimmunity in a case of spontaneous hyp ogly caemia. J Jpn Diabetes Soc 1970; 13:312-320.

[2] Uchigata Y, Hirata SY, Iwamoto Y. Insulin autoimmune syndrome (Hirata Disease) : Epidemiology in Asia including Japan. Diabetol Int 2010; 1: 21-25.

[3] Lupsa BC, Chong AY, Cochran EK, Soos MA, Semple RK, Gorden P. Autoimmune forms of hypoglycaemia. Medicine (Baltimore) 2009; 88:141-153.

[4] Bresciani E, Bussi A, Bazzigaluppi E, Balestrieri G. Insulin autoimmune syndrome induced by $\alpha$-lipoic acid in a Caucasian woman: Case report. Diabetes Care 2011; 34: e146.

[5] Annese S, Fadini G, Maran A. Caso di ipoglicemia autoimmune trattata con plasmaferesi. G It Diabetol Metab 2008; 28: 162-164.

[6] Cryer P. Glucose homeostasis and hypoglycemia. In: Kronenberg H, Melmed S, Polonsky K, Larsen P, eds. Williams Textbook of Endocrinology, 11th ed. Philadelphia, Saunders, Elsevier Inc. 2008: 1503-1533.

[7] Uchigata Y, Hirata Y, Omori Y, Iwamoto Y, Tokunaga K. Worldwide differences in the incidence of insulin autoimmune syndrome (Hirata Disease) with respect to the evolution of HLA-DR4 alleles. Human Immunol 2000; 61: 154-157

[8] Redmon B, Pyzdrowski KL, Elson MK, Kay NE, Dalmasso AP, Nuttall FQ. Brief report: hypoglycemia due to a monoclonal insulin-binding antibody in multiple myeloma. $\mathrm{N}$ Engl J Med 1992; 326: 994-998

[9] Seregni E, Ferrari L, Stivanello M Dogliotti L. Laboratory tests for neuroendocrine tumours. Q J Nucl Med 2000; 44:22-41

[10] Kim HS, Lee TY, Kim EY, et al. Fulminant type 1 diabetes in a pregnant woman a san initial manifestation of the insulin autoimmune syndrome. Diabet Med 2012 (in press).

[11] Zhang L, Nakayama M, Eisenbarth GS. Insulin as an autoantigen in NOD/Human diabetes. Curr Opin Immunol 2008; 20: 111-118.

[12] Ivanova M, Spassova $P$, Michailova A, Naumova E. Distributions of HLA class I alleles and haplotypes in Bulgarians - contribution to understanding the origin of the population. Tissue Antigens 2001; 57: 208-215.

[13] Barker JM. Clinical review: Type 1 diabetes-associated autoimmunity: natural history, genetic associations, and screening. J Clin Endocrinol Metab 2006; 91:1210-1217.

[14] Knip M, Korhonen S, Kulmala P, et al. Prediction of type 1 diabetes in the general population. Diabetes Care 2010; 33: 1206-1212. 